\title{
Shift-Orthogonal Wavelet Bases
}

\author{
Michael Unser, Senior Member, IEEE, Philippe Thévenaz, and Akram Aldroubi, Member, IEEE
}

\begin{abstract}
Shift-orthogonal wavelets are a new type of multiresolution wavelet bases that are orthogonal with respect to translation (or shifts) within one level but not with respect to dilations across scales. In this paper, we characterize these wavelets and investigate their main properties by considering two general construction methods. In the first approach, we start by specifying the analysis and synthesis function spaces and obtain the corresponding shift-orthogonal basis functions by suitable orthogonalization. In the second approach, we take the complementary view and start from the digital filterbank. We present several illustrative examples, including a hybrid version of the Battle-Lemarié spline wavelets. We also provide filterbank formulas for the fast wavelet algorithm. A shiftorthogonal wavelet transform is closely related to an orthogonal transform that uses the same primary scaling function; both transforms have essentially the same approximation properties. One experimentally confirmed benefit of relaxing the interscale orthogonality requirement is that we can design wavelets that decay faster than their orthogonal counterpart.
\end{abstract}

\section{INTRODUCTION}

$\mathbf{T}$ THE THEORY of the wavelet transform has resulted so far in three primary types of multiresolution bases of $L_{2}$ (the space of square integrable functions) [1]-[5]. The earliest ones were the orthogonal wavelet bases (e.g., Daubechies and Battle-Lemarié wavelets), which were fully characterized by Mallat [1], [6]. The second closely related family are the semiorthogonal wavelets, which span the same multiresolution subspaces as before but are not necessarily orthogonal with respect to shifts. The versatility of semi-orthogonal wavelet bases allows the introduction of many interesting properties [7]-[9] and almost any desirable shape [10] while retaining the orthogonality property across scales inherent to Mallat's construction. The third category is the biorthogonal wavelets, which are constructed using two multiresolution analyzes of $L_{2}$ instead of one, as in the previous cases [11]-[13]. Their advantage is that the wavelet filters can be shorter; in particular, they can be both FIR and linear phase, which typically is impossible otherwise.

One way to distinguish these various wavelet bases is to look at their orthogonality properties (Table I). This classification leads naturally to the identification of one more type, which has been neglected so far. These are the so-called shiftorthogonal wavelets, which are orthogonal to their translates

Manuscript received May 4, 1996; revised December 3, 1997. The associate editor coordinating the review of this paper and approving it for publication was Dr. Truong Q. Nguyen.

M. Unser is with the Biomedical Imaging Group, Swiss Federal Institute of Technology, EPFL, Lausanne, Switzerland (e-mail: Michael.Unser@epfl.ch).

P. Thévenaz is with the Biomedical Imaging Group, Swiss Federal Institute of Technology, EPFL, Lausanne, Switzerland.

A. Aldroubi is with the Department of Mathematics, Vanderbilt University, Nashville, TN 37240 USA.

Publisher Item Identifier S 1053-587X(98)04419-5.
TABLE I

Classification of Multiresolution Wavelet Bases

\begin{tabular}{|c|c|c|c|}
\hline & & \multicolumn{2}{|c|}{ intra-scale orthogonality } \\
\hline & & yes & no \\
\hline inter-scale & yes & orthogonal & semi-orthogonal \\
\hline orthogonality & no & shift-orthogonal & bi-orthogonal \\
\hline
\end{tabular}

within the same level but not across scales. We started the investigation of this class of transforms with the construction of a specific example using splines; it was presented in a preliminary report [14].

The purposes of this paper are to characterize these new wavelet bases in a systematic manner and to study their properties. We will consider two alternative construction methods, each interesting in its own right because of the particular insights that it provides. The first approach, which is developed in Section III, starts from two multiresolution analyses of $L_{2}$ and constructs the shift-orthogonal basis functions by suitable orthogonalization. The corresponding filterbank algorithm is presented in Section IV. The second construction method, which is developed in Section V, uses the reverse perspective and starts with a specification of the filters for the reconstruction algorithm.

In many ways, the shift-orthogonal wavelet construction methods that we propose may be thought of as a hybridization process between two orthogonal wavelet transforms. The resulting transform inherits most of the properties of the primary multiresolution on the synthesis side. In particular, the primary scaling function will control the rate of decay of the approximation error as the function of scale (order of the transform) as well as its asymptotic magnitude (i.e, the magnitude of the error for functions that are slowly varying with respect to the grid size) [15], [16]. Since the influence of the analysis space on global performance is marginal, we can use the freedom provided by the use of a second (analysis) multiresolution to design wavelets with a faster decay than their primary orthogonal counterparts. In other words, we can reduce the size of the wavelet synthesis filter while essentially preserving orthogonality.

Notation and Operators: $L_{2}$ is the vector space of real measurable, square-integrable functions $f(x), x \in R$. The corresponding $L_{2}$-inner product is $\langle f, g\rangle=\int_{-\infty}^{+\infty} f(x) g(x) d x$; the Fourier transform of $f$ is denoted by $\hat{f}(\omega)=$ $\int_{-\infty}^{+\infty} f(x) e^{-j \omega x} d x$.

$l_{2}$ is the space of square summable real-valued sequences (or discrete signals) $a(k), k \in Z$. The $z$-transform of $a$ is denoted 
TABLE II

Basic Discrete Signal Processing Operators AND Their EFFeCt IN THE Fourier Domain

\begin{tabular}{lll}
\hline \hline Opcrator & Notation & Fouricr transform \\
\hline Convolution & $(a * b)(k)=\sum_{l \in \mathcal{L}} a(l) b(k-l)$ & $\hat{a}(\omega) \cdot \hat{b}(\omega)$ \\
Time-shift & $a\left(k-k_{0}\right)=\left(\delta_{k_{1}} * a\right)(k)$ & $e^{-j\left(\omega k_{0}\right.} \cdot \hat{a}(\omega)$ \\
Time-reversal & $a^{T}(k)=a(-k)$ & $\bar{a}(\omega)$ \\
Up-sampling & {$[a]_{\uparrow_{2}}(k)$} & $\hat{a}(2 \omega)$ \\
Down-sampling & {$[a]_{\downarrow_{2}}(k)$} & $\left.\frac{1}{2}(\hat{a}(\omega) / 2)+\hat{a}(\omega / 2+\pi)\right)$ \\
Convolution inverse & $(a)^{-1}(k)$ & $1 / \hat{a}(\omega)$ \\
Convolution square-root & $(a)^{1 / 2}$ & $\sqrt{a}(\omega)$ \\
Modulation & $a^{ \pm}(k)=(-1)^{k} a(k)$ & $\hat{a}(\omega+\pi)$ \\
\hline
\end{tabular}

by a capital letter $A(z)=\sum_{k \in Z} a(k) z^{-k}$; the corresponding Fourier transform is $\hat{a}(\omega):=\sum_{k \in Z} a(k) e^{-j \omega k}=A\left(e^{j \omega}\right)$. We will consider a number of operators acting on discrete signals; these are summarized in Table II.

\section{PREliminary Notions}

We start with a brief review of fundamental notions in the theory of the wavelet transform and provide our own perspective on some of the basic concepts.

\section{A. Scaling Functions and Multiresolution Analyses}

Often, a scaling function is defined indirectly through the specification of its refinement filter $h$. Then, we have to worry about the delicate mathematical issues of the convergence of the iterated filter bank and the denseness of the wavelet representation in $L_{2}$ [2], [5]. Here, we prefer to start with a more explicit definition that takes care of these problems at the outset.

Definition 2.1: $\varphi(x)$ is an $L$ th-order scaling function iff it satisfies three conditions:

i)

$$
0<A \leq \hat{a}_{\varphi}(\omega)=\sum_{k \in Z}|\hat{\varphi}(\omega+2 \pi k)|^{2}<B<+\infty
$$

ii)

$$
\varphi(x / 2)=\sqrt{2} \sum_{k \in Z} h(k) \varphi(x-k)
$$

iii)

$$
\begin{aligned}
\hat{\varphi}(0) & =1 \quad \text { and } \quad \hat{\varphi}^{(m)}(2 \pi k)=0 \\
k & \in Z, \quad k \neq 0, \quad m=0, \ldots, L-1
\end{aligned}
$$

where $\hat{\varphi}(\omega)$ is the Fourier transform of $\varphi$, and $\hat{\varphi}^{(m)}(2 \pi k)$ is the $m$ th derivative of $\hat{\varphi}(\omega)$ evaluated at $2 \pi k$.

Condition i) ensures that $\varphi$ generates a Riesz basis and that the basic function space

$$
V_{0}(\varphi)=\left\{\sum_{k \in Z} c(k) \varphi(x-k), c \in l_{2}\right\}
$$

is a well-defined (closed) subspace of $L_{2}$. The constants $A$ and $B$ are the Riesz bounds of $\varphi$; in particular, the basis is orthonormal if and only if $A=B=1$.

Conditon ii) is the two-scale relation, which is the key to the multiresolution analysis. It allows us to define the coarse-tofine sequence of embedded subspaces $\{0\} \subset \cdots \subset V_{i}(\varphi) \subset$ $\cdots \subset V_{1}(\varphi) \subset V_{0}(\varphi) \subset V_{-1}(\varphi) \subset \cdots \subset L_{2}$ such that $f_{i}(x) \in V_{i}(\varphi) \Leftrightarrow f_{i}\left(2^{i} x\right) \in V_{0}(\varphi)$.

Condition iii) expresses a more technical order property, which is further discussed below. The minimum requirement for all wavelet constructions is $L=1$; this is also equivalent to the partition of unity condition $\sum_{k \in Z} \varphi(x-k)=1$ (the connection is given by Poisson's summation formula). Not only is this first-order property necessary for the convergence of the iterated filterbank [2], but it also guarantees that we can approximate any $L_{2}$-function as closely as we wish by choosing a scale that is sufficiently small [16]. Hence, the multiresolution decomposition is dense in $L_{2}$ and satisfies all the necessary requirements for the construction of wavelet bases [6].

\section{B. Order Properties}

Condition iii) comes from approximation theory and provides the most general definition of an $L$ th-order function [17], [18]. However, most wavelet textbooks use a simpler factorization condition on the transfer function of the refinement filter $h$

$$
H(z)=\left(\frac{1+z}{2}\right)^{L} \cdot Q(z)
$$

where $Q(z)$ is the transfer function of a stable filter with $Q(1)=\sqrt{2}$. In general, (5) is a stronger requirement than (3). Both conditions are equivalent if $\varphi$ (or $h$ ) is compactly supported.

The order conditions (3) and (5) are at the heart of wavelet theory. They have some remarkable consequences such as the vanishing moments of wavelets, the ability of the scaling function to reproduce polynomials of degree $n=L-1$, and the special eigenstructure of the two-scale transition operator (cf., [5, Ch. 7]). There are also lesser known approximation theoretic consequences that are quite relevant to our purpose. For an $L$-th order decomposition, the Strang-Fix theory implies that the approximation error at a given scale decays like the $L$ th power of that scale [17], [18]. In particular, when the function $f$ to approximate is sufficient smooth with respect to the current scale $a=2^{i}$, we have the asymptotic relation (cf., [16])

$$
\left\|f-f_{a}\right\|_{L_{2}}=C_{\varphi} \cdot a^{L} \cdot\left\|f^{(L)}\right\|_{L_{2}}+O\left(a^{L+1}\right)
$$

where $f_{a}$ denotes the approximation of $f$ at the scale $a$, $\left\|f^{(L)}\right\|_{L_{2}}$ is the $L_{2}$-norm of the $L$ th derivative of $f$, and $C_{\varphi}$ is the easily computable constant. The asymptotic limit is the same whether the projection is orthogonal or not, and the magnitude of $C_{\varphi}$ depends primarily on the smoothness of the synthesis scaling function $\varphi$ [16]. Interestingly, the spline constants are the smallest among all known wavelets of a given order $L$, whereas the Daubechies constants are the 
worst. In fact, the differences in magnitude are such that the performance of Daubechies' wavelets is no better than that of splines at half the resolution [15], [16]. The downside of splines is that the orthogonal Battle-Lemarié wavelets have poor decay. This motivates us to investigate ways in which we can improve their localization by being less stringent in enforcing orthogonality.

\section{From MultiRESOlution ANALYSES to WAVELETS}

In this section, we will start with two arbitrary multiresolution analyses $L_{2}$ and show how to obtain the corresponding shift-orthogonal wavelet transform. Our definition of such transforms is that the scaling function and wavelet on the synthesis side are orthogonal with respect to shifts within a given scale. We will characterize the corresponding basis functions and wavelets explicitly to establish the following result in a constructive manner.

Proposition 3.1: For any given two biorthogonal multiresolution analyses of $L_{2}$, there always exists a corresponding stable shift-orthogonal wavelet decomposition.

By stable decomposition, we mean that the shift-orthogonal wavelets provide an unconditional (or Riesz) basis of $L_{2}$. Note that the analysis and synthesis multiresolution spaces are specified independently of each other using two primary scaling functions $\varphi_{1}$ and $\varphi_{2}$ that are not necessarily biorthogonal to start with. Our only constraint for a consistent biorthogonal scheme is that the cosine of the angle between the basic analysis and synthesis spaces [see (10) below] must be strictly positive.

\section{A. Construction of Shift-Orthogonal Scaling Functions}

Let $\varphi_{1}$ and $\varphi_{2}$ be any two admissible analysis and synthesis scaling functions, respectively. We also define the following correlation sequences:

$$
\begin{array}{r}
a_{i j}(k)=\left\langle\varphi_{i}(x-k), \varphi_{j}(x)\right\rangle=\left(\varphi_{i}^{T} * \varphi_{j}\right)(k) \\
(i, j=1,2) .
\end{array}
$$

The Riesz basis condition i) implies that

$$
\begin{aligned}
& A_{1} \leq \hat{a}_{11}(\omega) \leq B_{1} \\
& A_{2} \leq \hat{a}_{22}(\omega) \leq B_{2}
\end{aligned}
$$

where the Riesz bounds $\left(A_{1}, B_{1}\right)$ and $\left(A_{2}, B_{2}\right)$ are all strictly positive and finite. The angle $\theta_{12}$ between the analysis and synthesis spaces $V_{i}\left(\varphi_{1}\right)$ and $V_{i}\left(\varphi_{2}\right)$ is given by (cf., [19])

$$
\cos \theta_{12}=\operatorname{ess} \inf _{\omega \in[0, \pi)} \frac{\left|\hat{a}_{12}(\omega)\right|}{\sqrt{\hat{a}_{11}(\omega) \cdot \hat{a}_{22}(\omega)}} \leq 1 .
$$

Note that the (biorthogonal) projection into $V_{i}\left(\varphi_{2}\right)$ perpendicular to $V_{i}\left(\varphi_{1}\right)$ is well defined if and only if $\cos \theta_{12}>0$ [20] (that is, if $-\pi / 2 \leq \theta_{12} \leq \pi / 2$ ). Thus, our stability condition for a consistent biorthogonal scheme is

$$
A_{12} \leq\left|\hat{a}_{12}(\omega)\right| \leq B_{12}
$$

where $A_{12} \geq \sqrt{A_{1} A_{2}} \cos \theta_{12}>0$, and $B_{12} \leq \sqrt{B_{1} B_{2}} \cos$ $\theta_{12}<+\infty$.
We can now characterize the corresponding shift-orthogonal scaling functions. The first step is to construct the synthesis scaling function $\phi(x) \in V_{0}\left(\varphi_{2}\right)$, which is the orthogonalized version of $\varphi_{2}$. This is done as described in [9]

$$
\phi(x)=\sum_{k \in Z}\left(a_{22}\right)^{-1 / 2}(k) \varphi_{2}(x-k)
$$

where $\left(a_{22}\right)^{-1 / 2}(k) \stackrel{\text { Fourier }}{\longleftrightarrow} 1 / \sqrt{\hat{a}_{22}(\omega)}$ denotes the squareroot convolution inverse of the symmetric sequence $a_{22}(k)$ (Table II). Here, we will only consider the symmetrical squareroot inverse. In principle, there are many other equivalent solutions, which differ only in terms of a phase factor.

Once $\phi$ has been specified, we can determine the corresponding (unique) dual function $\tilde{\phi}(x) \in V_{0}\left(\varphi_{1}\right)$, which must satisfy the biorthogonality constraint $\langle\phi(x), \phi(x-k)\rangle=\delta[k]$, where $\delta[k]$ is the discrete unit impulse. This leads to the following characterization:

$$
\tilde{\phi}(x)=\sum_{k \in Z}\left(a_{22}\right)^{1 / 2} *\left(a_{12}^{T}\right)^{-1}(k) \varphi_{1}(x-k)
$$

where $\left(a_{12}^{T}\right)^{-1}(k)$ is the convolution inverse (cf., Table II) of the cross-correlation sequence $a_{21}(k)=a_{12}^{T}(k)=\left\langle\varphi_{2}(x-k)\right.$, $\left.\varphi_{1}(x)\right\rangle$. Note that all the required inverse filters in (12) and (13) are well-defined because of the stability conditions (9) and (11).

Unlike $\phi$, the dual function $\tilde{\phi}$ is not orthogonal to its own shifts. In fact, we can easily compute its sampled autocorrelation sequence

$$
\begin{aligned}
a_{\tilde{\phi}}(k) & =\langle\tilde{\phi}(x-k), \tilde{\phi}(x)\rangle \\
& =\left(a_{22} *\left(a_{12} * a_{12}^{T}\right)^{-1} * a_{11}\right)(k) .
\end{aligned}
$$

If we express this relation in the Fourier domain and use (10), we obtain the Riesz bound [cf., (1)]

$$
1 \leq \hat{a}_{\tilde{\phi}}(\omega)=\left(\frac{\hat{a}_{22}(\omega) \cdot \hat{a}_{11}(\omega)}{\left|\hat{a}_{12}(\omega)\right|^{2}}\right) \leq \frac{1}{\left(\cos \theta_{12}\right)^{2}}
$$

which provides an interesting geometrical connection: We have full orthogonality if and only if $\cos \theta_{12}=1$ or, equivalently, when $\varphi_{1}$ and $\varphi_{2}$ generate the same space.

Having defined these new basis functions, we can now write the projection $P_{i}$ of a function $f \in L_{2}$ into $V_{i}\left(\varphi_{2}\right)$ perpendicular to $V_{i}\left(\varphi_{1}\right)$ as

$$
P_{i} f(x)=\sum_{k \in Z}\left\langle f, \tilde{\phi}_{i, k}\right\rangle \phi_{i, k}
$$

where we use the standard short-form notation $\phi_{i, k}=$ $2^{-i / 2} \phi\left(x / 2^{i}-k\right)$.

\section{B. Construction of Shift-Orthogonal Wavelets}

In the sequel, we also use a short-form notation for the synthesis and analysis spaces: $V_{i}=V_{i}\left(\varphi_{2}\right)$ and $\tilde{V}_{i}=V_{i}\left(\varphi_{1}\right)$. Let $W_{i}$ be the complementary (synthesis) wavelet space of $V_{i}$ in $V_{i-1}$ perpendicular to $\tilde{V}_{i}$, i.e., $\tilde{V}_{i-1}=W_{i} \oplus V_{i}$ with $W_{i} \perp \tilde{V}_{i}$. Likewise, let $\tilde{W}_{i}$ denote the complementary (analysis) wavelet space of $\tilde{V}_{i}$ in $\tilde{V}_{i-1}$ perpendicular to $V_{i}$, i.e., $\tilde{V}_{i-1}=\tilde{W}_{i} \oplus \tilde{V}_{i}$ with $\tilde{W}_{i} \perp V_{i}$. It can be shown (cf., [21, 
Th. 2.1]) that these wavelet spaces and their corresponding oblique projection operators are well defined if and only if the stability conditions (8), (9), and (11) are satisfied.

We now consider the construction of the corresponding shift-orthogonal wavelets at resolution level $i=1$. The synthesis wavelet $\psi(x / 2) \in W_{1}$ must satisfy the following conditions:

i) $\psi(x / 2) \in V_{0}$

ii) $\left\langle\psi(x / 2), \varphi_{1}(x / 2-k)\right\rangle=0$ because $\psi(x / 2)$ is perpendicular to $\tilde{V}_{1}$

iii) $\left\langle 2^{-1 / 2} \psi(x / 2), 2^{-1 / 2} \psi(x / 2-k)\right\rangle=\delta[k]$ because of the intra-scale orthogonality requirement.

It turns out that there is a function $\psi$, which again is unique up to a phase factor, that verifies all those conditions; it is given by

$$
\psi(x / 2)=\sum_{k \in Z}[p]_{\uparrow 2} * q(k) \varphi_{2}(x-k)
$$

where the sequences $q$ and $p$ are defined as

$$
\begin{aligned}
q(k+1) & =(-1)^{k} \cdot\left(h_{1}^{T} * a_{12}\right)(k) \\
p(k) & =\sqrt{2} \cdot\left(\left[q * q^{T} * a_{22}\right]_{\downarrow 2}\right)^{-1 / 2}(k) .
\end{aligned}
$$

The corresponding signal processing operators are defined in Table II. Note that this wavelet is nothing more than the orthogonalized version of the basic wavelet defined in [13], which is given by (17) with $p=$ identity. The Riesz bound conditions on the basic wavelet imply that the operator $p$ is a well-defined convolution operator from $l_{2}$ into $l_{2}$.

The dual analysis wavelet $\tilde{\psi}(x / 2) \in \tilde{W}_{1}$ must satisfy a similar set of conditions:

iv) $\tilde{\psi}(x / 2) \in \tilde{V}_{0}$;

v) $\left\langle\vec{\psi}(x / 2), \varphi_{2}(x / 2-k)\right\rangle=0$ because $\tilde{\psi}(x / 2)$ is perpendicular to $V_{1}$;

vi) $\left\langle 2^{-1 / 2} \tilde{\psi}(x / 2), 2^{-1 / 2} \psi(x / 2-k)\right\rangle=\delta[k]$, which expresses the biorthogonality condition.

After some algebraic manipulations, we obtain an expression that is similar to (17)-(19)

$$
\tilde{\psi}(x / 2)=\sum_{k \in Z}[\tilde{p}]_{\uparrow 2} * \tilde{q}(k) \varphi_{1}(x-k)
$$

where

$$
\begin{aligned}
\tilde{q}(k+1) & =(-1)^{k} \cdot\left(h_{2}^{T} * a_{12}^{T}\right)(k) \\
\tilde{p}(k) & =2 \cdot\left(p *\left[q^{T} * \tilde{q} * a_{12}^{T}\right]_{\downarrow 2}\right)^{-1}(k)
\end{aligned}
$$

with $p$ and $q$ defined in (19) and (18). Note that these equations can be simplified further, as described in the Appendix [cf., (A1), (A2)]. Here too, the fact that the angle between the analysis and synthesis spaces $\tilde{V}_{0}$ and $V_{0}$ is less than $\pi / 2$ in absolute value (stability condition) ensures that the convolution and square-root inverses in (22) and (19) are well posed and that the resulting digital filters $p$ and $\tilde{p}$ are stable and invertible.

It is also of interest to determine the Riesz bounds for the dual wavelet $\tilde{\psi}(x)$, which is not orthogonal. For this purpose,
TABLE III

$\cos \theta_{12}$ Between the Splines Space $V_{0}\left(\varphi^{n_{1}}\right)$ and $V_{0}\left(\varphi^{n_{2}}\right)$

\begin{tabular}{c|c|c|c|c|c|c|c|c}
\hline \hline$n_{1} \quad n_{2}$ & 0 & 1 & 2 & 3 & 4 & 5 & 6 & 7 \\
\cline { 2 - 9 } 0 & 1 & 0 & 0.8729 & 0 & 0.8162 & 0 & 0.7837 & 0 \\
\hline 1 & & 1 & 0 & 0.9709 & 0 & 0.9374 & 0 & 0.9103 \\
\hline 2 & & & 1 & 0 & 0.9857 & 0 & 0.9634 & 0 \\
\hline 3 & & & & 1 & 0 & 0.9911 & 0 & 0.9751 \\
\hline 4 & & & & & 1 & 0 & 0.9939 & 0 \\
\hline 5 & & & & & & 1 & 0 & 0.9955 \\
\hline 6 & & & & & & & 1 & 0 \\
\hline 7 & & & & & & & & 1 \\
\hline
\end{tabular}

we must compute the sampled autocorrelation sequence

$$
a_{\tilde{\psi}}(k)=\left\langle 2^{-1 / 2} \tilde{\psi}(x / 2-k), 2^{-1 / 2} \tilde{\psi}(x / 2)\right\rangle .
$$

After a series of manipulations similar to the one given in the Appendix, we find that

$$
a_{\tilde{\psi}}(k)=\left(a_{22} *\left(a_{12} * a_{12}^{T}\right)^{-1} * a_{11}\right)(k) .
$$

By going to the Fourier domain, we obtain

$$
\begin{aligned}
A_{\tilde{\psi}} & =1 \leq \hat{a}_{\tilde{\psi}}(\omega) \\
& =\left(\frac{\hat{a}_{22}(\omega) \cdot \hat{a}_{11}(\omega)}{\left|\hat{a}_{12}(\omega)\right|^{2}}\right) \leq B_{\tilde{\psi}}=\frac{1}{\left(\cos \theta_{12}\right)^{2}}
\end{aligned}
$$

which is the same as for the dual analysis function [cf., (15)]. This result also reflects the property that the angle between the wavelet spaces $W_{i}$ and $\tilde{W}_{i}$ is the same as the angle between $V_{i}$ and $\tilde{V}_{i}$.

\section{Example: Shift-Orthogonal Spline Wavelets}

As an illustrative example, we consider the case $\varphi_{1}(x)=$ $\varphi^{n_{1}}(x)$ and $\varphi_{2}(x)=\varphi^{n_{2}}(x)$, where $\varphi^{n}$ is the noncentral (or causal) B-spline of degree $n$. Specifically, $\varphi^{n}$ is obtained from the $(n+1)$-fold convolution of the unit indicator function $\chi_{[1,0)}$ (or B-spline of degree 0 ). The spline spaces that are generated in this way are essentially the same as those in our first example with $n_{1}=1$ and $n_{2}$ odd [14]. A notable difference, however, is that the present basis functions are not centered about the origin. This modification is necessary if we want to include splines of even degree, which are not covered otherwise. The connection between both formulations is provided by the relation

$$
\varphi^{n}(x)=\beta^{n}\left(x-\frac{n+1}{2}\right)
$$

where $\beta^{n}(x)$ is the centered B-spline of degree $n$.

The causal B-spline functions are all valid scaling functions in the sense of Definition 2.1. Assuming that the cosine of the angle between the analysis and synthesis spaces is greater than zero, the shift-orthogonal basis functions and wavelets are then described explicitly by (12), (13), (17), and (20). What is still required is the determination of the refinement filters $h_{1}$ and $h_{2}$ and the correlation sequences $a_{11}, a_{22}$, and $a_{12}$.

Recalling that the refinement filter for a B-spline of degree 0 is $H^{0}(z)=\left(1+z^{-1}\right) / \sqrt{2}$ and that the $\mathrm{B}$-splines are 


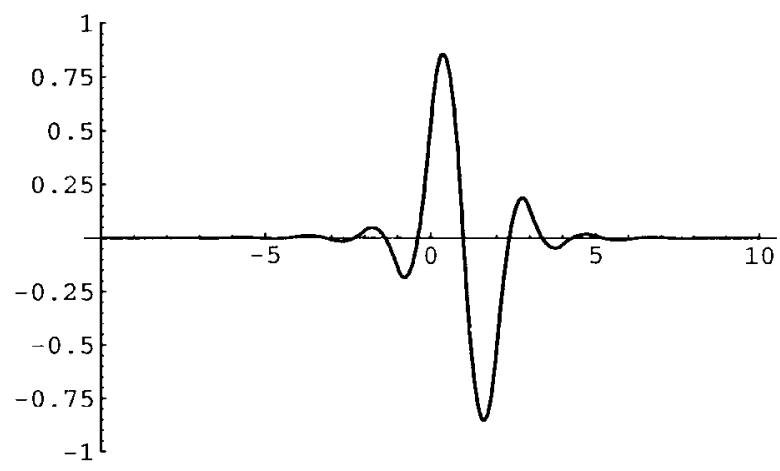

(a)

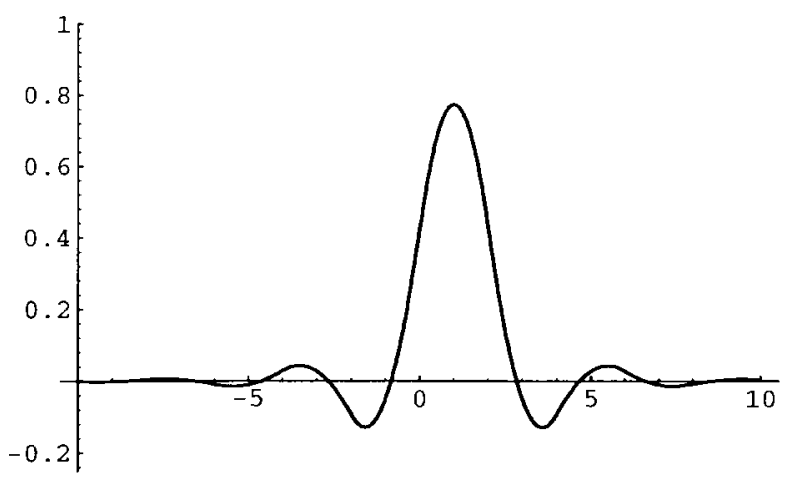

(c)

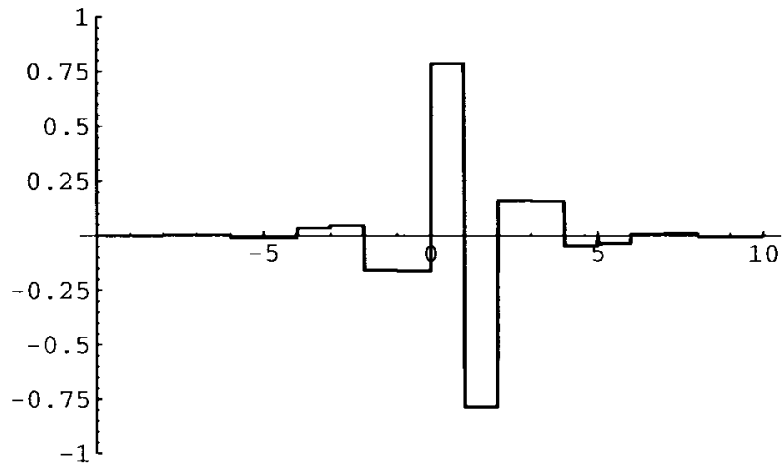

(b)

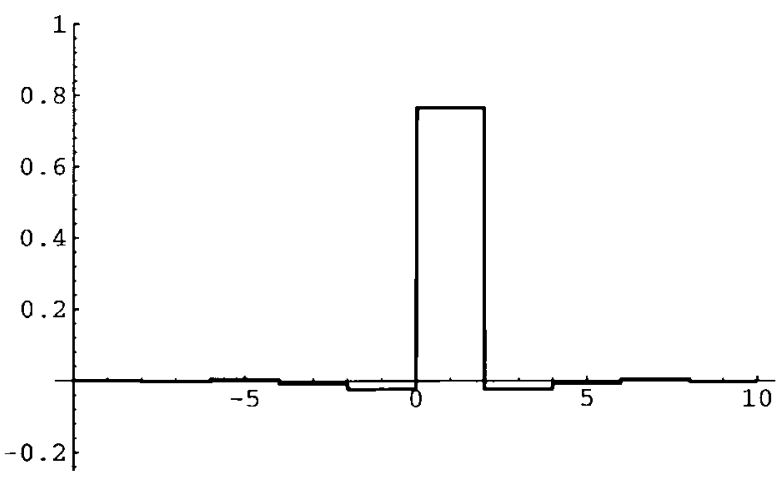

(d)

Fig. 1. Dual sets of quadratic spline and piecewise constant wavelets and scaling functions at the first resolution level. (a) Shift-orthogonal quadratic spline wavelet. (b) Dual piecewise constant wavelet. (c) Orthogonal quadratic spline Battle-Lemarié scaling function. (d) Dual piecewise constant analysis scaling function. The basis functions in (a) and (b) [resp., (c) and (d)] are quadratic splines with knots at the integers (resp., at the even integers).

generated by repeated convolution, it is not difficult to show that $h_{1}=h^{n_{1}}$ and $h_{2}=h^{n_{2}}$, where $h^{n}$ is the binomial filter of order $n+1$

$$
h^{n}(k) \stackrel{z}{\longleftrightarrow} H^{n}(z)=\sqrt{2} \cdot\left(\frac{1+z^{-1}}{2}\right)^{n+1} .
$$

This equation also shows that the approximation order of the corresponding wavelet transform is $L_{2}=n_{2}+1$ (cf., Section II-B). Likewise, we can deduce that the corresponding shift-orthogonal spline wavelet $\psi(x)$ has $L_{1}=n_{1}+1$ vanishing moments (cf., [2]). Next, we use the fact that the convolution between two B-splines of degree $n_{1}$ and degree $n_{2}$ is a B-spline of degree $n_{1}+n_{2}+1$, and we obtain the explicit form of the correlation sequences

$$
\begin{aligned}
a_{11}(k) & =\varphi^{2 n_{1}+1}\left(k+\frac{2 n_{1}+2}{2}\right)=\beta^{2 n_{1}+1}(k) \\
a_{22}(k) & =\varphi^{2 n_{2}+1}\left(k+\frac{2 n_{2}+2}{2}\right)=\beta^{2 n_{2}+1}(k) \\
a_{12}(k) & =\varphi^{n_{1}+n_{2}+1}\left(k+\frac{2 n_{2}+2}{2}\right) \\
& =\beta^{n_{1}+n_{2}+1}\left(k-\frac{\left(n_{2}-n_{1}\right)}{2}\right)
\end{aligned}
$$

which are all with finite impulse response (FIR). While the
Riesz bounds conditions (8) and (9) are satisfied for any degree $n$, the angle between the present spline spaces is less than $\pi / 2$ in absolute value (i.e., $\cos \theta_{12}>0$ ) only when $\left(n_{1}-n_{2}\right)$ is even, that is, when the degrees $n_{1}$ and $n_{2}$ are both odd or both even. Explicit values for all combinations of splines up to degree 7 are given in Table III. The various shift-orthogonal scaling functions and wavelets for the case $n_{1}=0$ and $n_{2}=2$ are shown in Fig. 1. We can observe that the basis functions are piecewise constant on the analysis side and piecewise quadratic with a first order of continuity on the synthesis side. Interestingly, the analysis function $\tilde{\phi}$ has a very fast decay and is reasonably close to a $\mathrm{B}$-spline of degree 0 .

In addition, note that for the particular case $n_{1}=n_{2}$, the present construction yields the Battle-Lemarié spline wavelets, which are completely orthogonal [22], [23].

\section{SHIFT-ORTHOGONAL WAVELET TRANSFORM AND FILTERBANK ALGORITHM}

\section{A. Wavelet Expansions}

Because of our stability conditions, each wavelet space $W_{i}$ is a well-defined subspace of $L_{2}$, which admits $\left\{\psi_{i, k}=\right.$ $\left.2^{-i / 2} \psi\left(x / 2^{-i}-k\right)\right\}_{k \in Z}$ as an orthonormal basis. Since by definition $\cup_{i \in Z} V_{i}$ is dense in $L_{2}$, it follows that the set $\left\{\psi_{i, k}\right\}_{(i, k) \in Z^{2}}$ is an unconditional basis of $L_{2}$ and that every function $f \in L_{2}$ can be represented by its shift-orthogonal 
wavelet expansion

$$
f(x)=\sum_{i \in Z} \sum_{k \in Z}\left\langle f, \tilde{\psi}_{i, k}\right\rangle \psi_{i, k} .
$$

The special feature of this decomposition is that the basis functions are orthogonal with respect to shifts (index $k$ ) but not across scales or dilations (index $i$ ).

If we now consider the partial wavelet expansion up to the scale $i-1$, we obtain the projection of $f$ into $V_{i}$ perpendicular to $\tilde{V}_{i}$

$$
P_{i} f(x)=\sum_{j=+\infty}^{i-1} \sum_{k \in Z}\left\langle f, \tilde{\psi}_{j, k}\right\rangle \psi_{j, k}
$$

which can also be represented in terms of the scaling function at resolution $i$ [cf., (16)]. Note that the approximation error at scale $i$ in the shift-orthogonal case will always be slightly above the error of the corresponding orthogonal transform (least squares solution). Specifically, we have (cf., [19, Th. 3])

$$
\begin{aligned}
\forall f \in L_{2}, \quad\left\|f-P_{i}^{\perp} f\right\| & \leq\left\|f-P_{i} f\right\| \\
& \leq \frac{1}{\cos \theta_{12}}\left\|f-P_{i}^{\perp} f\right\|
\end{aligned}
$$

where $P_{i}^{\perp} f$ denotes the orthogonal projection of $f$ into $V_{i}$. One should note, however, that the upper bound on the right side of (32) corresponds to a worst-case scenario that is rarely encountered in practice. This can also be understood in geometric terms. Specifically, the true angle between $f-P_{i}^{\perp} f$ and $f-P_{i} f$ will usually be much smaller than $\theta_{12}$, which represents the maximum angle between the subspaces $V_{i}$ and $\tilde{V}_{i}$, both of infinite dimension.

Because the worst-case angles between the various spline spaces are relatively small (Table III), we can expect the shiftorthogonal spline wavelets to provide essentially the same type of energy compaction as the corresponding orthogonal Battle-Lemarié wavelet transforms. In other words, we will get essentially the same $L_{2}$ approximation error when we start discarding the smaller scale wavelet coefficients. It also appears that the hybrid cubic spline wavelet transform $\left(n_{1}=1\right.$ and $n_{2}=3$ ) first described in [14] is nearly orthogonal.

\section{B. Filterbank Algorithm}

We can implement the wavelet transform (30) or (31) iteratively by using a standard tree-structured perfect reconstruction filterbank [4]. The algorithm requires the specification of two analysis filters and two synthesis filters $(\tilde{h}, \tilde{g})$ and $(h, g)$, respectively. These are defined as

$$
\left\{\begin{array}{l}
\tilde{h}(k)=\frac{1}{\sqrt{2}}\langle\tilde{\phi}(x / 2), \phi(x+k)\rangle \\
\tilde{g}(k)=\frac{1}{\sqrt{2}}\langle\tilde{\psi}(x / 2), \phi(x+k)\rangle \\
h(k)=\frac{1}{\sqrt{2}}\langle\phi(x / 2), \tilde{\phi}(x-k)\rangle \\
g(k)=\frac{1}{\sqrt{2}}\langle\psi(x / 2), \tilde{\phi}(x-k)\rangle .
\end{array}\right.
$$

The easiest way to determine these filters is to perform the appropriate change of coordinate system and to express $\phi(x / 2)$ and $\psi(x / 2)$ (resp., $\tilde{\phi}(x / 2)$ and $\tilde{\psi}(x / 2))$ in terms of the integer shifts of $\phi$ (resp., $\tilde{\phi})$. This provides an explicit characterization of their impulse response in the signal domain. The most involved derivation is the computation of a simplified form of the wavelet analysis filter $\tilde{g}(k)$, which is given in the Appendix. We have chosen here to present frequency domain formulas because these are the most useful in practice. Our results are summarized as

$$
\begin{aligned}
& H\left(e^{j \omega}\right):=\hat{h}(\omega)=\hat{h}_{2}(\omega) \cdot \sqrt{\frac{\hat{a}_{22}(\omega)}{\hat{a}_{22}(2 \omega)}} \\
& \tilde{H}\left(e^{j \omega}\right):=\hat{\tilde{h}}(\omega)=\overline{\hat{h}_{1}(\omega)} \cdot \frac{\hat{a}_{12}(\omega)}{\hat{a}_{12}(2 \omega)} \cdot \sqrt{\frac{\hat{a}_{22}(2 \omega)}{\hat{a}_{22}(\omega)}} \\
& G\left(e^{j \omega}\right):=\hat{g}(\omega)=\frac{1}{\sqrt{2}} e^{-j \omega} \cdot \overline{\hat{h}_{1}(\omega+\pi)} \cdot \hat{p}(2 \omega) \\
& \text { - } \hat{a}_{12}(\omega+\pi) \cdot \sqrt{\hat{a}_{22}(\omega)} \\
& \tilde{G}\left(e^{j \omega}\right):=\hat{\tilde{g}}(\omega)=e^{j \omega} \cdot \frac{\sqrt{2} \cdot \hat{h}_{2}(\omega+\pi)}{\hat{a}_{12}(2 \omega) \sqrt{\hat{a}_{22}(\omega)}} \cdot \frac{1}{\hat{p}(2 \omega)}
\end{aligned}
$$

where the auxiliary filters $\tilde{p}(2 \omega)$ and $\hat{q}_{a}(\omega)$ are defined as

$$
\begin{aligned}
& \hat{p}(2 \omega)=\frac{2}{\sqrt{\hat{q}_{a}(\omega)+\hat{q}_{a}(\omega+\pi)}} \\
& \hat{q}_{a}(\omega)=\left|\hat{h}_{1}(\omega+\pi)\right|^{2} \cdot\left|\hat{a}_{12}(\omega+\pi)\right|^{2} \cdot \hat{a}_{22}(\omega) .
\end{aligned}
$$

Note that the equation for $H$ is the same as that given by Mallat for the orthogonal case simply because both transforms share the same (orthogonal) synthesis scaling function $\phi$. This filter satifies the condition

$$
H(z) H(1 / z)+H(-z) H(-1 / z)=2
$$

and is called a quadrature mirror filter (QMF). If the scaling functions $\varphi_{1}$ and $\varphi_{2}$ are compactly supported (as in the spline case), it can be shown that all filters decay exponentially fast. In general, they will not be FIR.

In order to compute a truncated version of a filter's impulse response, the simplest approach is to evaluate its transfer function at the discrete frequencies $\omega_{i}=2 \pi i / N, i=0, \ldots, N-1$, where $N$ is chosen sufficiently large to avoid aliasing in the signal domain. The impulse response is then determined by using an $N$-point inverse FFT. The first 15 filter coefficients for the example in Fig. 1 (quadratic splines) are given in Table IV. The lowpass filter $h$ is the same as the corresponding quadratic Battle-Lemarié filter. Interestingly, the wavelet synthesis filter $g$ decays significantly faster and turns out to be similar to the Haar filter $G^{0}(z)=\left(1-z^{-1}\right) / \sqrt{2}$.

A crucial practical issue is the decay of the various filters. A dispersion index that can be computed easily is the standard time-localization measure

$$
\Delta_{h}=\frac{\left\|\left(k-x_{h}\right) h(k)\right\|_{l_{2}}}{\|h(k)\|_{l_{2}}}
$$

where $\|h\|_{l_{2}}$ denotes the $l_{2}$-norm of the filter $h$, and $x_{h}$ represents the center of its impulse response. When the response is a two-sided exponential of the form $h(k)=C \cdot \alpha^{\left|k-k_{0}\right|}$, we can derive the relation

$$
|\alpha|=\frac{\sqrt{\Delta_{h}^{2}+1-\sqrt{1+2 \Delta_{h}^{2}}}}{\Delta_{h}}
$$


TABLE IV

IIR Filter COEFFicients FOR THE ShIFT-Orthogonal Quadratic SPline WaVelet Transform With $n_{1}=0$ AND $n_{2}=2$

\begin{tabular}{|c|c|c|c|c|}
\hline$k$ & $\bar{h}$ & $\tilde{g}$ & $h$ & $g$ \\
\hline$-k<$ & $\tilde{h}(-k)=\tilde{h}(k-1)$ & $\tilde{g}(-k)=-\tilde{g}(k-1)$ & $h(-k)=h(k+1)$ & $g(-k)=-g(k+1)$ \\
\hline$\dddot{-1}$ & 0.729915 & 0.702088 & 0.137963 & 0.0130307 \\
\hline 0 & 0.729915 & 0.702088 & 0.68037 & -0.706862 \\
\hline 1 & 0.00766485 & 0.138748 & 0.68037 & 0.706862 \\
\hline 2 & -0.0195685 & 0.136703 & 0.137963 & -0.0130307 \\
\hline 3 & -0.0112333 & -0.0322905 & -0.124835 & -0.0129547 \\
\hline 4 & -0.00645937 & -0.0368606 & -0.0207472 & 0.0019878 \\
\hline 5 & 0.00460937 & 0.00809514 & 0.0419821 & 0.00223624 \\
\hline 6 & 0.00537476 & 0.0100018 & 0.00423816 & 0.000308145 \\
\hline & -0.00215218 & -0.00223964 & -0.0151129 & 0.000232297 \\
\hline & -0.00225881 & -0.00265455 & -0.00112345 & 0.0000522267 \\
\hline & 0.000779012 & 0.000586975 & 0.00570553 & 0.0000488927 \\
\hline & 0.000888193 & 0.000716251 & 0.000334821 & $8.69216 \mathrm{E}-06$ \\
\hline 11 & -0.000289915 & -0.000159667 & -0.00221175 & $8.51609 \mathrm{E}-06$ \\
\hline & -0.000323867 & -0.000190971 & -0.000107847 & $1.47356 \mathrm{E}-06$ \\
\hline 13 & 0.000102918 & 0.0000423399 & 0.000873158 & $1.57089 \mathrm{E}-06$ \\
\hline 14 & 0.00011698 & 0.0000513501 & 0.0000364102 & $2.52536 \mathrm{E}-07$ \\
\hline 15 & & & -0.000349114 & $2.88225 \mathrm{E}-07$ \\
\hline 16 & & & & \\
\hline
\end{tabular}

TABLE V

Time Localization of THE Four Filters fOR VARIOUS SHIFT-ORTHOGONAL WAVELET TRANSFORMS

\begin{tabular}{lllll}
\hline \hline$\left(n_{1}, n_{2}\right)$ & \multicolumn{1}{c}{$\Delta_{h}$} & $\Delta_{\ddot{g}}$ & $\Delta_{h}$ & $\Delta_{g}$ \\
\hline \multicolumn{2}{c}{ Design method I (hybrid splines) : } & & \\
$(0,0)$ & 0.5 & 0.5 & 0.5 & 0.5 \\
$(0,2)$ & 0.513712 & 0.787392 & 0.787688 & 0.50299 \\
$(0,4)$ & 0.546779 & 1.01545 & 1.01074 & 0.506707 \\
$(1,1)$ & 0.646834 & 0.646834 & 0.646834 & 0.646834 \\
$(1,3)$ & 0.656403 & 0.909417 & 0.906014 & 0.650565 \\
$(1,5)$ & 0.675763 & 1.11805 & 1.1058 & 0.652716 \\
\hline Design method II : & & & \\
$(0,2)$ & 0.514936 & 0.788199 & 0.787688 & 0.5 \\
\hline
\end{tabular}

which can be used to estimate the exponential decay parameter $\alpha$ without performing any curve fitting. The corresponding time constant is then given by $\tau_{\alpha}=-1 / \log |\alpha|$. We considered all four filters and performed explicit numerical computations of $\Delta_{h}$ for spline wavelet transforms up to degree 5 ; the results are presented in Table V. Note that the third measurement $\left(\Delta_{h}\right)$ also provides the dispersion index for the corresponding Battle-Lemarié transform $\left(n=n_{2}\right)$, whose filters are all derived from a single QMF template that is precisely $h$ (cf., subsection $\mathrm{C}$ below). While the localizations of the filters $h$ and $\tilde{g}$ are comparable, it appears that our hybridization scheme has effectively reduced the decay of the filters $g$ and $\tilde{h}$. The shortest filter is the wavelet synthesis filter $(g)$, which has approximately the same localization as a Battle-Lemarié filter of degree $n=n_{1}$, and this almost independently of $n_{2}$. Thus, by varying the order of the analysis and synthesis spaces, we can control the decay of the various digital filters (which tend to be associated in pairs).

\section{Filter Properties}

We have already observed that the filter $h$ is the same as in the orthogonal case. Similarly, the shift-orthogonality property imposes constraints on the wavelet synthesis filter $g$.
Proposition 4.1: For a shift-orthogonal wavelet transform of $L_{2}$, the synthesis filters $H(z)$ and $G(z)$ are both QMF [cf., (40)], with the lowpass and highpass conditions

$$
\begin{aligned}
& H(1)=\sqrt{2} \Leftrightarrow H(-1)=0 \\
& G(1)=0 \Leftrightarrow G(-1)=\sqrt{2} .
\end{aligned}
$$

Proof: The scaling function $\phi$ can be written as

$$
2^{-1 / 2} \phi(x / 2)=\sum_{k \in Z} h(k) \phi(x-k)
$$

and the lowpass synthesis filter $h$ automatically has the right properties because $\phi$ is an orthogonal scaling function (cf., [1]). Likewise, the wavelet $\psi$ can be represented as

$$
2^{-1 / 2} \psi(x / 2)=\sum_{k \in Z} g(k) \phi(x-k)
$$

where $g$ is the wavelet synthesis filter. The shift-orthogonality condition implies that

$$
\left\langle 2^{-1 / 2} \psi(x / 2), 2^{-1 / 2} \psi(x / 2-k)\right\rangle=\delta[k] .
$$

Replacing $\psi$ by its expression (46) and using the orthogonality of $\phi$, we get

$$
\left[g * g^{T}\right]_{\downarrow 2}(k)=\delta[k] .
$$

The corresponding relation in the $z$-transform domain is precisely the QMF condition

$$
\frac{1}{2}(G(z) G(1 / z)+G(-z) G(-1 / z))=1 .
$$

Because $\varphi_{1}$ (or $\tilde{\phi}$ ) satisfies the partition of unity condition, $\psi$ has at least one vanishing moment $\int \psi(x) d x=0$ (i.e., $\psi$ is an admissible wavelet). Since $\sqrt{2} \int \psi(x) d x=$ $\sum_{k \in Z} g(k) \int \phi(x) d x$ and $\int \phi(x) d x \neq 0$, this implies that $\sum_{k \in Z} g(k)=G(1)=0$.

\section{From Synthesis Filters to WAVELETS}

We will now investigate the complementary approach and construct shift-orthogonal wavelet transforms by starting with the filterbank. The method that is described next is based on Proposition 4.1. Its only potential problem is that not all perfect reconstruction filterbanks generate admissible scaling functions and wavelets for $L_{2}$. In particular, we need to make sure a posteriori that the underlying scaling functions, which are now defined through an infinite recursion, converge to well-defined limits in $L_{2}$ [5].

We propose the following design procedure: Select two quadrature mirror filter templates $H_{a}(z)$ (index $a$ for analysis) and $H_{s}(z)$ (index $s$ for synthesis) of order $L_{a}$ and $L_{s}$, respectively, which are such that their dyadic spectral coherence function $^{1}$

$$
\Lambda_{a s}(z)=\frac{1}{2}\left(H_{s}(z) H_{a}\left(z^{-1}\right)+H_{s}(-z) H_{a}\left(-z^{-1}\right)\right)
$$

is nonvanishing on the unit circle. Note that $\left|\Lambda_{a s}\left(e^{j \omega}\right)\right| \leq 1$ as a result of the Schwarz inequality (cf., [24]). As we shall see, the parameters $L_{a}$ and $L_{s}$ control the order of the analysis and

\footnotetext{
${ }^{1} \Lambda_{a s}(z)$ is the $z$-transform of $\left[h_{a}^{T} * h_{s}\right]_{\downarrow 2 \uparrow 2}(k)$, which is the even index part of the cross-correlation between $h_{a}$ and $h_{s}$.
} 


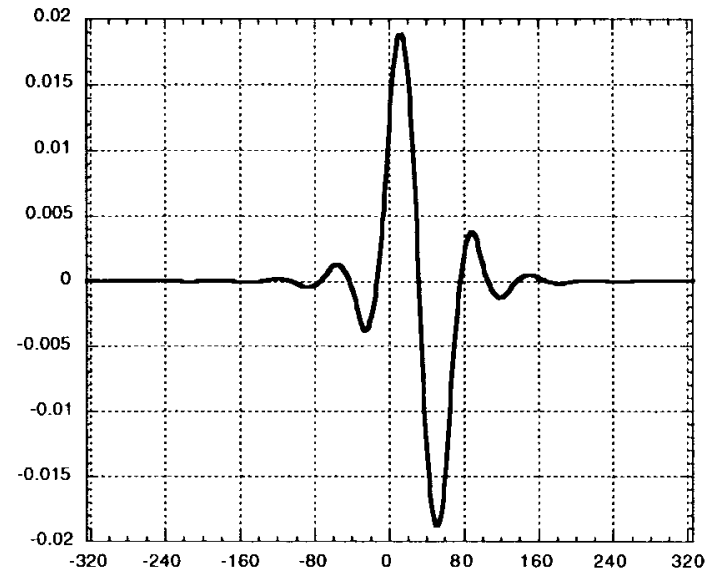

(a)

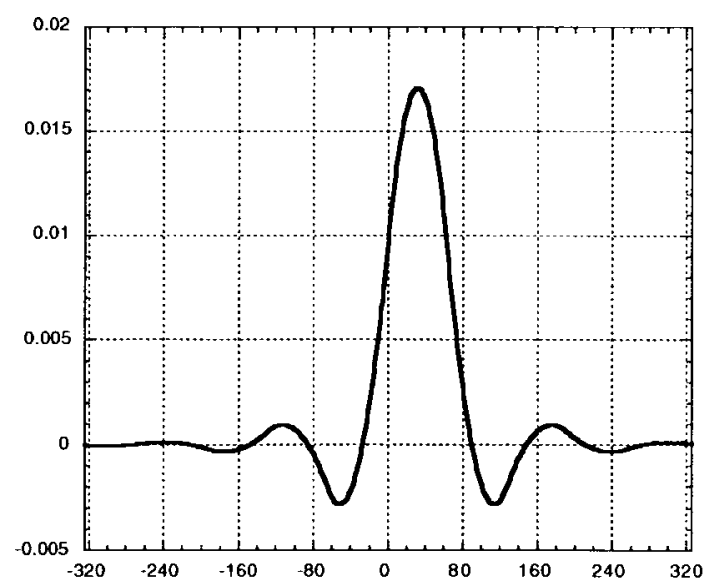

(c)

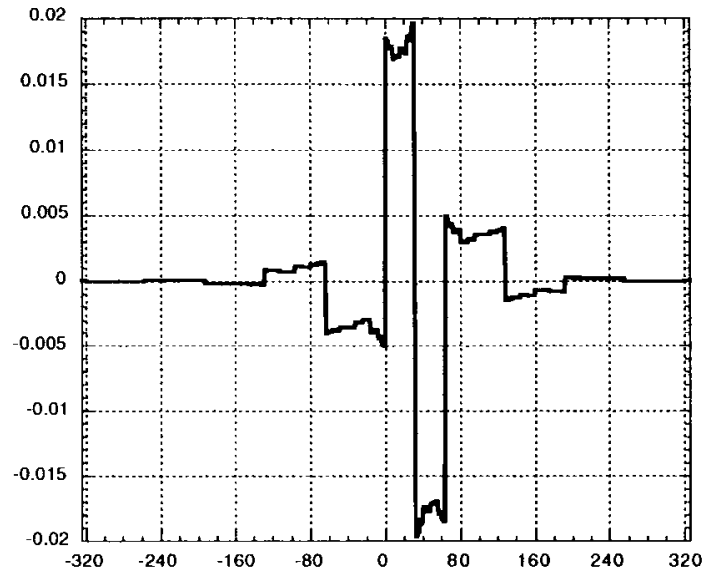

(b)

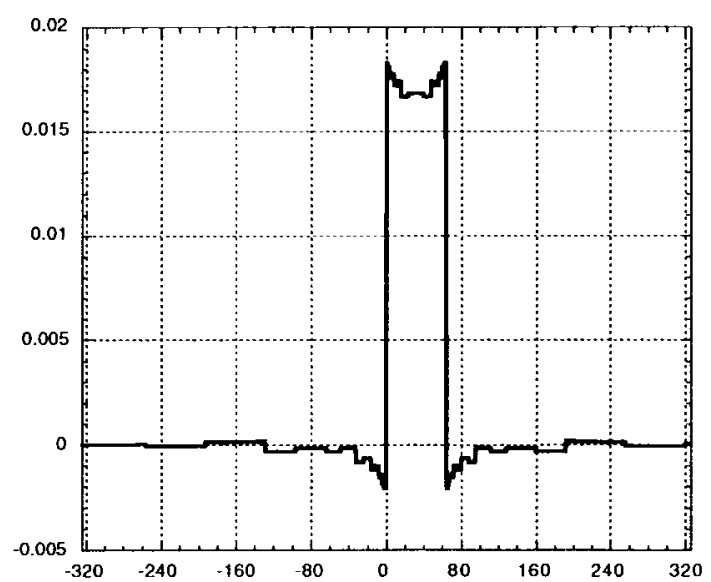

(d)

Fig. 2. Dual sets of wavelets [(a) and (b)] and scaling functions [(c) and (d)] associated with the filterbank coefficients in Table VI at the sixth resolution level. These graphs were generated using six scale iterations of the filterbank algorithm. The synthesis function (c) is the same as the quadratic spline scaling function $\phi(x)$ in Fig. 1; the wavelet (a) is also a quadratic spline that is very similar (but not identical) to the function $\psi(x)$ in Fig. 1. The analysis functions in (b) and (d), on the other hand, are no longer piecewise constant.

synthesis spaces $V_{0}(\tilde{\phi})$ and $V_{0}(\phi)$, respectively. In particular, the lowpass condition $H_{a}(1)=H_{s}(1)=\sqrt{2}$ (i.e., $L_{a} \geq 1$ and $L_{s} \geq 1$ ) is necessary for the convergence of the iterated filterbank (cf., [2]).

These templates are then used to specify the lowpass and highpass synthesis filters

$$
\begin{aligned}
& H(z)=H_{s}(z) \\
& G(z)=-z \cdot H_{a}\left(-z^{-1}\right)
\end{aligned}
$$

where the latter is the time-reversed, shifted, and modulated version of the analysis template $H_{a}(z)$. With this particular setting, we are obviously satisfying the conditions in Proposition 4.1. In addition, note that (49) implies that the wavelet filter $G\left(e^{j \omega}\right)$ has a zero of multiplicity $L_{a}$ at the origin $\omega=0$. This, in turn, implies that the shift-orthogonal wavelet $\psi(x)$ has $N_{a}$ vanishing moments.

We can now write down the perfect reconstruction conditions for the filterbank algorithm (cf., [4])

$$
\left\{\begin{array}{l}
\tilde{H}(z) H(z)+\tilde{G}(z) G(z)=2 \\
\tilde{H}(z) H(-z)+\tilde{G}(z) G(-z)=0
\end{array}\right.
$$

and solve these equations to determine the remaining analysis filters. The determinant of the system is

$$
\begin{aligned}
\Delta(z) & =H(z) G(-z)-H(-z) G(z) \\
& =z \cdot\left(H_{s}(z) H_{a}\left(z^{-1}\right)+H_{a}(-z) H_{a}\left(-z^{-1}\right)\right) \\
& =2 z \cdot \Lambda_{a s}(z)
\end{aligned}
$$

and the solution is

$$
\begin{aligned}
& \tilde{H}(z)=\frac{2 G(-z)}{\Delta(z)}=\frac{H_{a}\left(z^{-1}\right)}{\Lambda_{a s}(z)} \\
& \tilde{G}(z)=\frac{-2 H(-z)}{\Delta(z)}=\frac{z^{-1} \cdot H_{s}(-z)}{\Lambda_{a s}(z)} .
\end{aligned}
$$

Since the determinant is nonvanishing, both analysis filters are stable and well defined. For the same reason, the analysis filter $\tilde{h}$ will inherit the order properties of $H_{a}(z)$, and the analysis space $V_{0}(\tilde{\phi})$ will have an order of approximation $L_{a}$. Likewise, the wavelet filter $\tilde{G}\left(e^{j \omega}\right)$ will have an $L_{s}$ thorder zero at the origin, which implies that the corresponding dual analysis $\tilde{\psi}$ has $L_{s}$ vanishing moments. This again reflects the fact that the synthesis space $V_{0}(\phi)$ has an $L_{s}$ th order of approximation. The rational form of transfer functions in (52) and (53) also suggests that it is impossible to have FIR shiftorthogonal analysis filters unless $\Lambda_{a s}(z)=1$, in which case, $H_{a}(z)=H_{s}(z)$, and the transformation is orthogonal. 
TABLE VI

Filter Coefficients For the ShifT-Orthogonal Hybrid WaVElet Transform (HAar/BATTLE-LeMarié QMF with $n_{2}=2$ )

\begin{tabular}{lllll}
\hline \hline$k$ & \multicolumn{1}{c}{$\tilde{h}$} & \multicolumn{1}{c}{$\tilde{g}$} & \multicolumn{1}{c}{$h$} & $g$ \\
\hline$-k<0$ & $h(-k)=h(k-1)$ & $\tilde{g}(-k)=-\tilde{g}(k-1)$ & $h(-k)=h(k+1)$ & \\
$\ldots$ & $\ldots$ & $\ldots$ & $\ldots$ & \\
-1 & 0.735505 & 0.707107 & 0.137963 & \\
0 & 0.735505 & -0.707107 & 0.68037 & 0.707107 \\
1 & -0.00710868 & 0.139234 & 0.68037 & -0.707107 \\
2 & -0.00710868 & 0.139234 & 0.137963 & \\
3 & -0.0114306 & -0.0352308 & -0.124835 & \\
4 & -0.0114306 & -0.0352308 & -0.0207472 & \\
5 & 0.00611006 & 0.00875716 & 0.0419821 & \\
6 & 0.00611006 & 0.00875716 & 0.00423816 & \\
7 & -0.00241844 & -0.00218759 & -0.0151129 & \\
8 & -0.00241844 & -0.00218759 & -0.00112345 & \\
9 & 0.00087672 & 0.000545397 & 0.00570553 & \\
10 & 0.00087672 & 0.000545397 & 0.000334821 & \\
11 & -0.000307468 & -0.000136101 & -0.00221175 & \\
12 & -0.000307468 & -0.000136101 & -0.000107847 & \\
13 & 0.000106857 & 0.000033947 & 0.000873158 & \\
14 & 0.000106857 & 0.000033947 & 0.0000364102 & \\
15 & $\ldots$ & $\ldots$ & -0.000349114 & \\
16 & $\ldots$ & $\ldots$ & & \\
\hline
\end{tabular}

The proposed construction method can be used to combine the filterbanks associated with different orthogonal wavelet transforms (e.g., Daubechies or splines). With this hybridization technique, the underlying approximation space $V_{0}(\phi)$ is the same as the one used in the orthogonal case. The analysis space, on the other hand, will differ because of the determinant term in (52). As before, it is usually preferable to put a low order on the analysis side and a higher order on the synthesis side.

As a design example, we have considered mixing the spline QMF's for $n_{1}=0$ and $n_{2}=2$, which is very similar to the shift-orthogonal construction in Section III-C, except that we are now starting from the filters. The motivation for using $H_{a}(z)=\left(1+z^{-1}\right) / \sqrt{2}$ is that it is the shortest admissible QMF. The corresponding filter coefficients obtained by inverse FFT are given in Table VI. Not too surprisingly, they are quite similar to those in Table IV. The filter localization measures are also given at the bottom of Table IV and should be compared with those of the first design method. The corresponding scaling functions and wavelet graphs were generated through an iterative filtering process and are shown in Fig. 2. While the synthesis functions are very similar to those in Fig. 1 (quadratic splines), the functions on the analysis side are no longer piecewise constant. In fact, they have lost some regularity because of the presence of the $\Lambda_{a s}(z)$ term in the denominator of (52), which causes $\hat{\tilde{\phi}}(\omega)$ to decay at a slower rate than $1 / \omega$. Based on our simulations, it appears that $\hat{\tilde{\phi}}(\omega)$ still has sufficient decay for $\tilde{\phi}(x)$ to be in $L_{2}$, but the limit is discontinuous.

\section{CONCLUSION}

In this paper, we have characterized the class of shiftorthogonal wavelet transforms and presented two general construction methods. In contrast with previous semi- and biorthogonal constructions, we have only relaxed the orthogonality constraint in-between resolution levels. As a result, the basis functions are still orthogonal within a given wavelet channel (or scale). The first way to think of shift-orthogonal wavelets is as a particularization of the general biorthogonal case, which uses two different multiresolution analyzes of $L_{2}$. In terms of constraints, the situation is very much analogous to the construction of orthogonal wavelets by orthogonalization of semiorthogonal basis functions such as splines. An example that permits a very direct visualization of the two underlying biorthogonal subspaces (piecewise constant functions versus quadratic splines) is provided in Fig. 1. Shift-orthogonal wavelets can also be thought of as hybrids that are obtained by combining two orthogonal wavelet transforms. The constraints are such that both synthesis filters will be QMF; however, they can be selected independently of each other. We have shown that the lowpass filter determines the approximation order of the representation, whereas the highpass filter controls the decay of the wavelet.

In practice, it would make sense to use a higher order representation on the synthesis side and a lower order on the analysis side. In this way, we can use the degrees of freedom offered by the second (analysis) multiresolution to obtain faster decaying wavelets while essentially preserving the approximation and orthogonality properties of the primary higher order transform (e.g., Battle-Lemarié splines).

\section{APPENDIX}

\section{DERIVATION OF THE WAVELET ANALYSIS FILTER}

First, we will simplify the expression of the dual wavelet, e.g., (20)-(22). For this purpose, we write the dual wavelet as

$$
\tilde{\psi}(x / 2)=\sum_{k \in Z} r(k) \varphi_{1}(x-k)
$$

where $r(k)=[\tilde{p}]_{\uparrow 2} * \tilde{q}(k)$, with $\tilde{p}$ and $\tilde{q}$ given by (21) and (22). Replacing these sequences by their explicit expressions, we get

$$
\begin{aligned}
r(k)= & 2 \cdot[p]_{\uparrow 2}^{-1} *\left[h_{1}^{ \pm} * h_{2}^{T \pm} * a_{21}^{ \pm} * a_{21}^{ \pm} * a_{21}\right]_{\downarrow 2 \uparrow 2}^{-1} \\
& * \delta_{1} * a_{21} * h_{2}^{T \pm}(k)
\end{aligned}
$$

using the operator notations in Table II. Since the sequence $a_{21} * a_{21}^{ \pm}(k)$ is zero for all odd integers and is left unchanged by the operator $[\cdot]_{\downarrow 2 \uparrow 2}$, we can factor it out, which yields

$$
\begin{aligned}
r(k)= & 2 \cdot[p]_{\uparrow 2}^{-1} *\left[h_{1}^{ \pm} * h_{2}^{T \pm} * a_{21}^{ \pm}\right]_{\downarrow 2 \uparrow 2}^{-1} *\left(a_{21}^{ \pm} * a_{21}\right)^{-1} \\
& * \delta_{1} * a_{21}^{ \pm} * h_{2}^{T \pm}(k) .
\end{aligned}
$$

Next, we notice that

$$
\left[h_{1}^{ \pm} * h_{2}^{T \pm} * a_{21}^{ \pm}\right]_{\downarrow 2}=\left[h_{1} * h_{2}^{T} * a_{21}\right]_{\downarrow 2}(k)=a_{21}(k)
$$

where the right-hand side follows from the two-scale relation for $\varphi_{21}(x)=\varphi_{2}^{T} * \varphi_{1}(x)$, which is easily derived by convolution. Finally, putting things together, we end up with

$$
r(k)=2 \cdot[p]_{\uparrow 2}^{-1} *\left[a_{21}\right]_{\uparrow 2}^{-1} *\left(a_{21}\right)^{-1} * \delta_{1} * h_{2}^{T \pm}(k) .
$$

Since the basic scaling function can also be represented by

$$
\varphi_{1}(x)=\sum_{k \in Z}\left(a_{22}\right)^{-1} * a_{21}(k) \tilde{\phi}(x-k)
$$

we can also rewrite $\tilde{\psi}$ as

$$
\widetilde{\psi}(x / 2)=\sqrt{2} \sum_{k \in Z} \tilde{g}^{T}(k) \tilde{\phi}(x-k)
$$

where the time-reversed version of the analysis filter is given 
by

$$
\tilde{g}^{T}(k)=\sqrt{2} \cdot[p]_{\uparrow 2}^{-1} *\left[a_{21}\right]_{\uparrow 2}^{-1} * \delta_{1} * h_{2}^{T \pm} *\left(a_{22}\right)^{-1}(k) .
$$

This is precisely the inverse Fourier transform of (37).

\section{ACKNOWLEDGMENT}

The authors would like to thank Dr. M. Vrhel for helping us to generate Fig. 2 and B. Bowman for the careful editing of the manuscript.

\section{REFERENCES}

[1] S. G. Mallat, "A theory of multiresolution signal decomposition: The wavelet representation," IEEE Trans. Pattern Anal. Machine Intell., vol. 11, pp. 674-693, 1989.

[2] I. Daubechies, Ten Lectures on Wavelets. Philadelphia, PA: SIAM, 1992.

[3] B. Jawerth and W. Sweldens, "An overview of wavelet based multiresolution analyzes," SIAM Rev., vol. 36, no. 3, pp. 377-412, Sept. 1994.

[4] M. Vetterli and J. Kovacević, Wavelets and Subband Coding. Englewood Cliffs, NJ: Prentice-Hall, 1995.

[5] G. Strang and T. Nguyen, Wavelets and Filter Banks. Wellesley, MA: Wellesley-Cambridge, 1996.

[6] S. G. Mallat, "Multiresolution approximations and wavelet orthogonal bases of $L^{2}(R)$," Trans. Amer. Math. Soc., vol. 315, no. 1, pp. 69-87, 1989.

[7] M. Unser, A. Aldroubi, and M. Eden, "On the asymptotic convergence of B-spline wavelets to Gabor functions," IEEE Trans. Inform. Theory, vol. 38, pp. 864-872, Mar. 1992.

[8] , "A family of polynomial spline wavelet transforms," Signal Process., vol. 30, no. 2, pp. 141-162, Jan. 1993.

[9] A. Aldroubi and M. Unser, "Families of multiresolution and wavelet spaces with optimal properties," Numer. Funct. Anal. Optimiz., vol. 14, nos. 5-6, pp. 417-446, 1993.

[10] P. Abry and A. Aldroubi, "Designing multiresolution analysis-type wavelets and their fast algorithms," J. Fourier Anal. Applicat., vol. 2, no. 2, pp. 135-159, 1995.

[11] A. Cohen, I. Daubechies, and J. C. Feauveau, "Bi-orthogonal bases of compactly supported wavelets," Commun. Pure Appl. Math., vol. 45, pp. 485-560, 1992

[12] M. Vetterli and C. Herley, "Wavelets and filter banks: Theory and design," IEEE Trans. Signal Processing, vol. 40, pp. 2207-2232, Sept. 1992.

[13] P. Abry and A. Aldroubi, "Semi- and bi-orthogonal MRA-type wavelet design and their fast algorithms," in Proc. SPIE Conf. Wavelet Applicat. Signal Image Process. III, San Diego, CA, July 9-14, 1995, pp. 452-463.

[14] M. Unser, P. Thę́venaz, and A. Aldroubi, "Shift-orthogonal wavelet bases using splines," IEEE Signal Processing Lett., vol. 3, pp. 85-88, Mar. 1996.

[15] W. Sweldens and R. Piessens, "Asymptotic error expansions for wavelet approximations of smooth functions II," Numer. Math., vol. 68, no. 3, pp. 377-401, 1994

[16] M. Unser, "Approximation power of biorthogonal wavelet expansions," IEEE Trans. Signal Processing, vol. 44, pp. 519-527, Mar. 1996.

[17] G. Strang and G. Fix, "A Fourier analysis of the finite element variational method," in Constructive Aspect of Functional Analysis, Rome, Italy: Edizioni Cremonese, 1971, pp. 796-830.

[18] G. Strang, "Wavelets and dilation equations: A brief introduction," SIAM Rev., vol. 31, pp. 614-627, 1989.

[19] M. Unser and A. Aldroubi, "A general sampling theory for nonideal acquisition devices," IEEE Trans. Signal Processing, vol. 42, pp. 2915-2925, Nov. 1994.

[20] A. Aldroubi, "Oblique projections in atomic spaces," in Proc. Amer. Math. Soc., vol. 124, no. 7, pp. 2051-2060, July 1996.

[21] A. Aldroubi, P. Abry, and M. Unser, "Construction of biorthogonal wavelets starting from any two given multiresolution analyzes," IEEE Trans. Signal Processing, vol. 46, pp. 1130-1133, Apr. 1998.

[22] G. Battle, "A block spin construction of ondelettes. Part I: Lemarié functions," Commun. Math. Phys., vol. 110, pp. 601-615, 1987.

[23] P.-G. Lemarié, "Ondelettes à localization exponentielles," J. Math. Pures Appl., vol. 67, no. 3, pp. 227-236, 1988.

[24] A. Aldroubi and M. Unser, "Oblique projections in discrete signal subspaces of $l_{2}$ and the wavelet transform," in Proc. Math. Imaging: Wavelet Applicat. Signal Image Process. II, San Diego, CA, 1994, pp. 36-45.

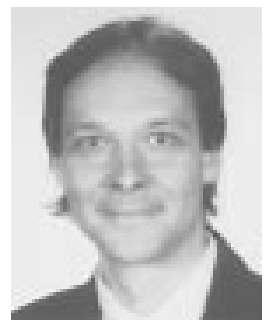

Michael Unser (M'89-SM'94) was born in Zug, Switzerland, on April 9, 1958. He received the M.S. (summa cum laude) and Ph.D. degrees in electrical engineering in 1981 and 1984, respectively, from the Swiss Federal Institute of Technology, Lausanne, Switzerland.

From 1985 to 1997 , he was with the Biomedical Engineering and Instrumentation Program, National Institutes of Health, Bethesda, MD, where he had been heading the Image Processing Group. He is now Professor of Biomedical Image Processing at the Swiss Federal Institute of Technology. His research interests include the application of image processing and pattern recognition techniques to various biomedical problems, multiresolution algorithms, wavelet transforms, and the use of splines in signal processing. He is the author of over 70 published journal papers in these areas.

Dr. Unser serves as an Associate Editor for the IEEE Signal Processing LETTERS and is a member of the Image and Multidimensional Signal Processing Committee of the IEEE Signal Processing Society. He is also on the editorial boards of Signal Processing, The Journal of Visual Communication and Image Representation, and Pattern Recognition and was a former Associate Editor (1992-1995) for the IEEE Transactions on IMAGE Processing. He co-organized the 1994 IEEE-EMBS Workshop on Wavelets in Medicine and Biology and serves as conference chair for SPIE's Wavelet Applications in Signal and Image Processing, which has been held annually since 1993. He received the Dommer Prize for excellence from the Swiss Federal Institute of Technology in 1981, the Research Prize of the Brown-Boveri Corporation (Switzerland) for his thesis in 1984, and the IEEE Signal Processing Society's 1995 Best Paper Award (in the IMDSP technical area) for a paper with A. Aldroubi and M. Eden on B-spline signal processing.

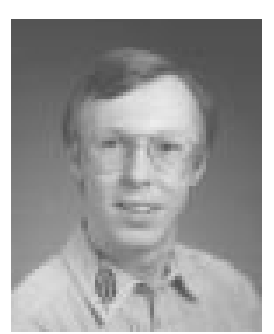

Philippe Thévenaz was born in 1962 in Lausanne, Switzerland. He graduated from the Swiss Federal Institute of Technology (EPFL), Lausanne, in January 1986 with a diploma in microengineering. He received the Ph.D. degree in June 1993 from the Institute of Microtechnology (IMT), University of Neuchâtel, Neuchâtel, Switzerland, with a thesis on the use of the linear prediction residue for textindependent speaker recognition.

At IMT, he worked as research assistant and scientist, first in the domain of image processing (optical flow) and then in the domain of speech processing (speech coding and speaker recognition). He then worked as a Visiting Fellow with the Biomedical Engineering and Instrumentation Program, National Institutes of Health (NIH), Bethesda, MD, where he developed research interests that include splines and multiresolution signal representations, geometric image transformations, and biomedical image registration. Since 1998, he has been with EPFL as first assistant.

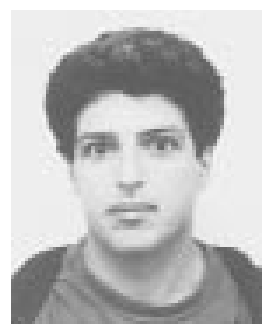

Akram Aldroubi (M'88) received the M.S. degree in electrical engineering from the Swiss Federal Institute of Technology, Lausanne, Switzerland, in 1982. He received the M.S. and Ph.D. degrees in mathematics from Carnegie Mellon University, Pittsburgh, PA, in 1984 and 1987, respectively.

$\mathrm{He}$ is currently an Associate Professor of Mathematics at Vanderbilt University, Nashville, TN. His research interests include wavelet theory, sampling theory, signal and image processing, functional analysis, harmonic analysis, and mathematical biology.

Dr. Aldroubi is a member of the American Mathematical Society and the American Association of America. He received the Rene Cousin Prize for excellence from the Swiss Federal Institute of Technology in 1982 and the IEEE Signal Processing Society's 1995 Best Senior Paper Award (in the IMDSP technical area) for a paper with M. Unser and M. Eden on B-spline signal processing. He is on the editorial board of IEEE TRANSACTIONS ON Signal Processing, The Journal of Mathematical Imaging and Vision, The Journal of Fourier Analysis and Applications, and the International Journal on Pattern Recognition. He is also on the editorial board of several other technical book series. He is co-editor (with M. Unser) of the book Wavelet in Medicine and Biology (Boca Raton, FL: CRC, 1996) and co-editor (with E.B. Lin) of the recent book Wavelets, Multiwavelets, and Applications (Series in Contemporary Mathematics, New York: Amer. Math. Soc., 1998). 